\title{
Supporting Information for Calculation of Charge-Transfer Electronic Coupling with Nonempirically Tuned Range-Separated Density Functional
}

\author{
Hirotaka Kitoh-Nishioka ${ }^{\dagger, \ddagger, *}$, and Koji Ando ${ }^{\mathbf{\Phi}, *}$ \\ ${ }^{\dagger} J S T$, PRESTO, \\ 4-1-8 Honcho, Kawaguchi, Saitama 332-0012, Japan \\ ${ }^{\ddagger}$ Center for Compuational Sciences, University of Tsukuba, \\ 1-1-1 Tennodai, Tsukuba, Ibaraki, 305-8577, Japan \\ 'Department of Information and Sciences, Tokyo Woman's Christian University, \\ 2-6-1 Zenpukuji, Suginami-ku, Tokyo 167-8585, Japan \\ *E-mail: hkito@ccs.tsukuba.ac.jp; ando_k@lab.twcu.ac.jp \\ March 21, 2019
}



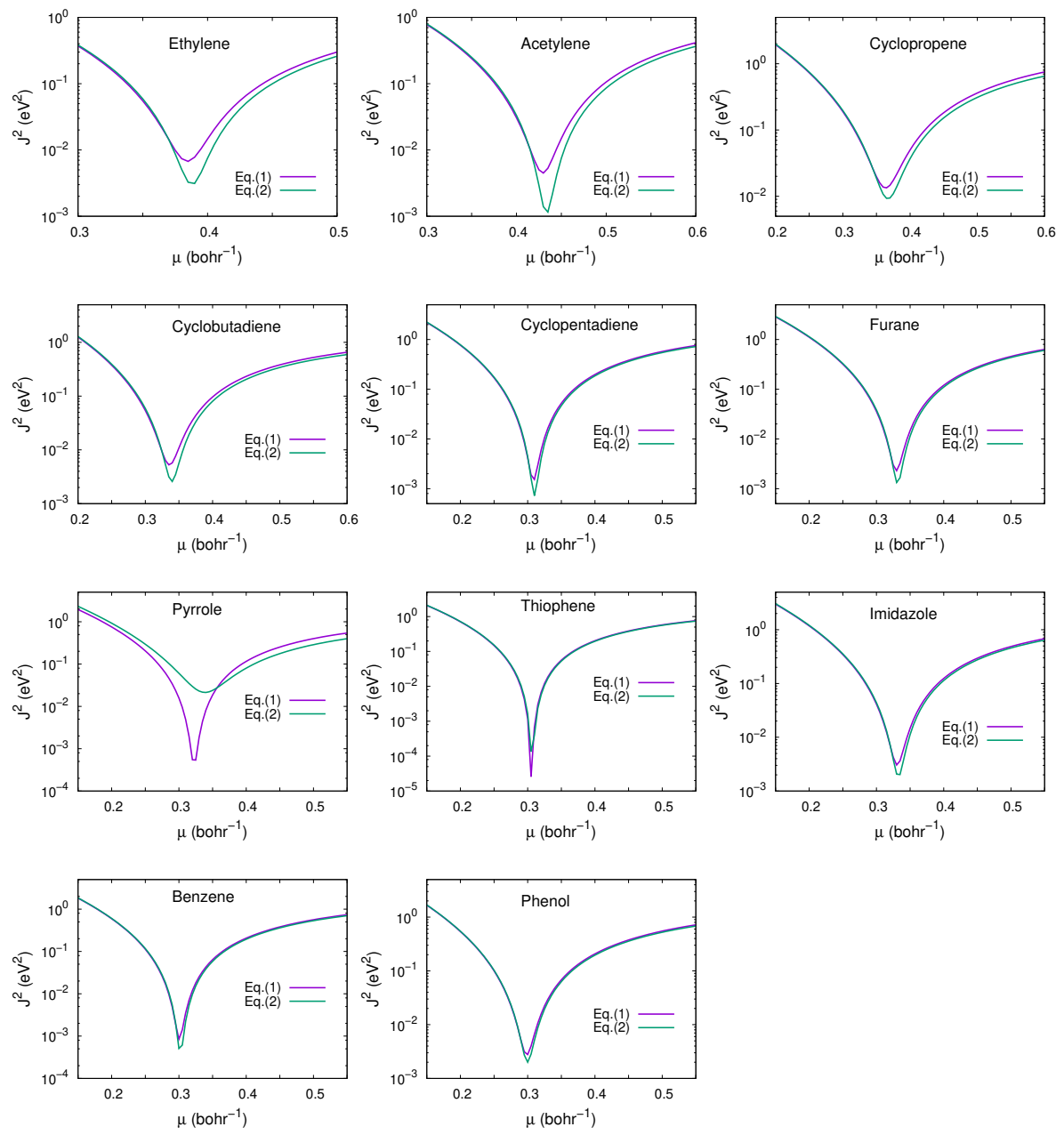

Fig.S1. $J_{\mathbf{H}}^{2}(\mu)$ and $J_{\mathbf{L}}^{2}(\mu)$ of Eqs. (1) and (2) for the molecules of the HAB11 set. 

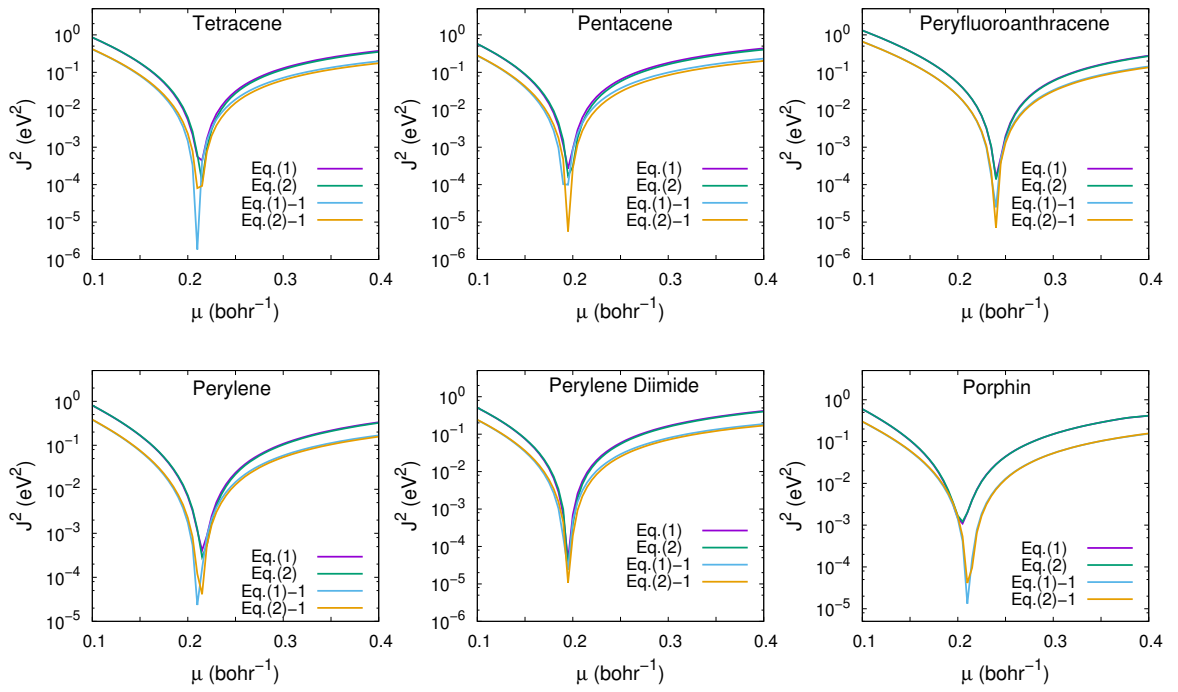

Fig.S2. Same as Fig. 1 for the other molecules of the HAB7- set than anthracene. 

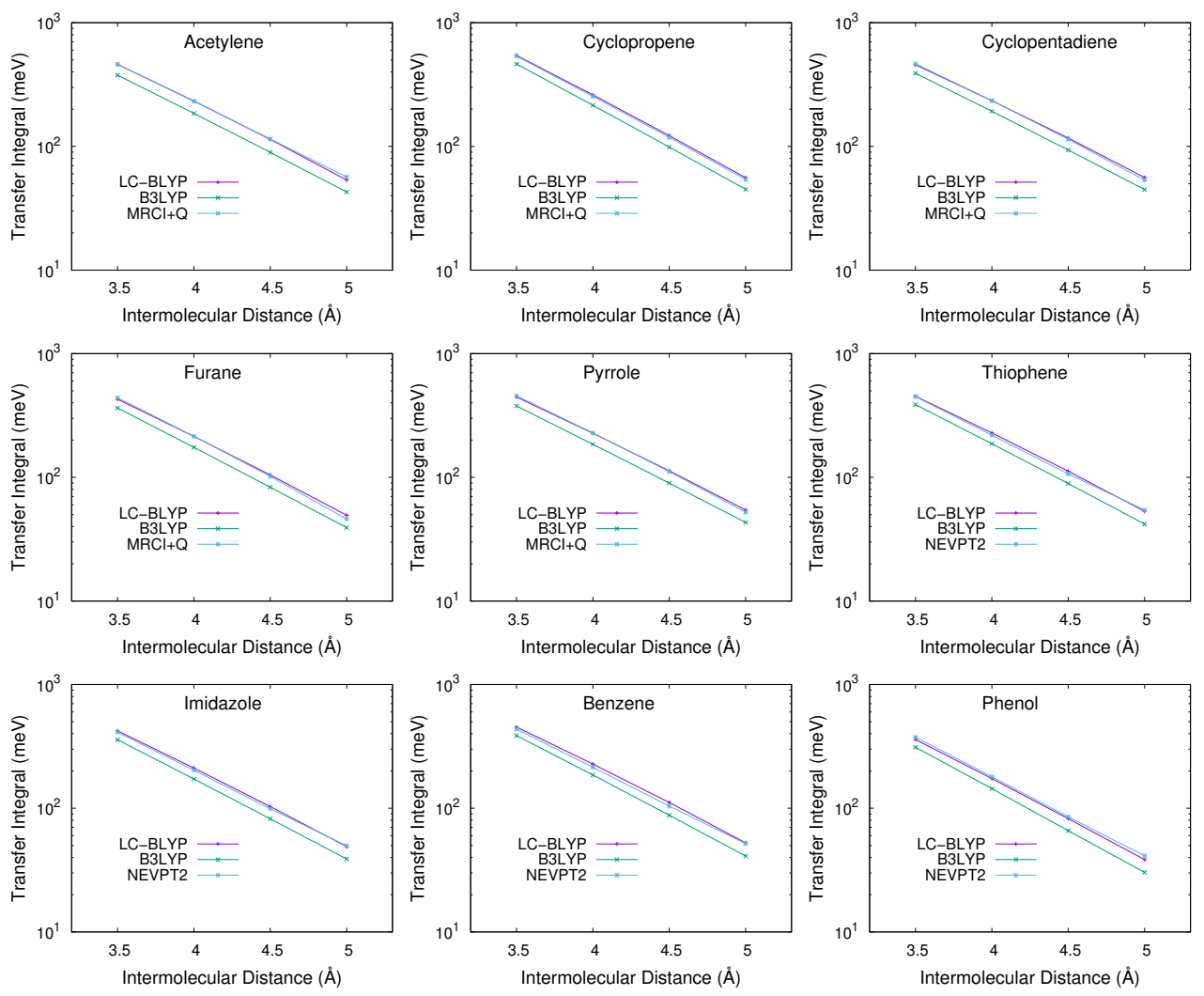

Fig.S3. $T_{\mathrm{DA}}^{\prime}(R)$ for the molecules of the HAB11 set other than ethylene and cyclobutadiene that are displayed in Fig. 4. 

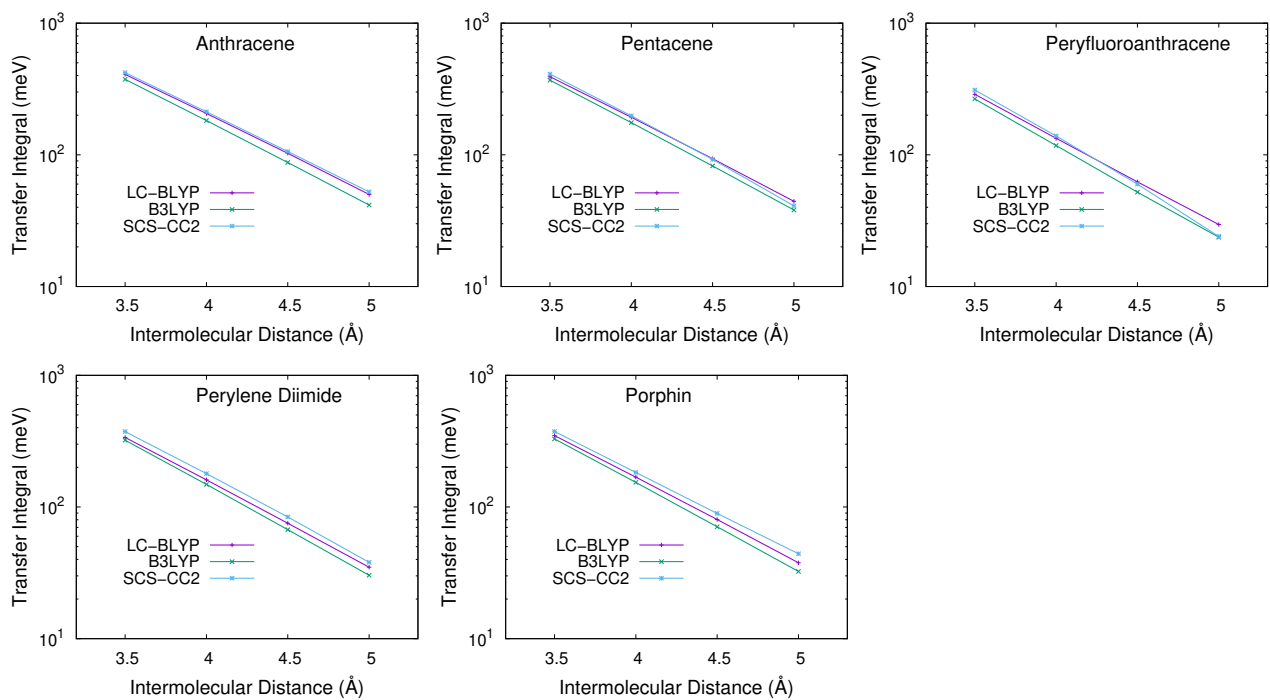

Fig.S4. $\quad T_{\mathrm{DA}}^{\prime}(R)$ for the molecules of the HAB7- set other than tetracene and perylene that are displayed in Fig. 4. 
Table S1. Optimized $\mu$ (in bohr ${ }^{-1}$ ) from Eqs.(1) and (2) without the first term, $\mu_{1}^{\prime}$ and $\mu_{2}^{\prime}$, compared with those from the full Eqs. (1) and (2), $\mu_{1}$ and $\mu_{2}$.

\begin{tabular}{lcccc}
\hline \hline Molecule & $\mu_{1}$ & $\mu_{2}$ & $\mu_{1}^{\prime}$ & $\mu_{2}^{\prime}$ \\
\hline Anthracene & 0.235 & 0.235 & 0.230 & 0.235 \\
Tetracene & 0.215 & 0.215 & 0.210 & 0.210 \\
Pentacene & 0.195 & 0.195 & 0.195 & 0.195 \\
Peryfluoroanthracene & 0.240 & 0.240 & 0.240 & 0.240 \\
Perylene & 0.215 & 0.215 & 0.210 & 0.215 \\
Perylene Diimide & 0.195 & 0.195 & 0.195 & 0.195 \\
Porphin & 0.205 & 0.205 & 0.210 & 0.210 \\
\hline \hline
\end{tabular}


Table S2. Electronic coupling energy for hole transfers in seven cation dimers of the HAB11 set calculated with another three RS and two hybrid density functionals.

\begin{tabular}{|c|c|c|c|c|c|c|}
\hline & & \multicolumn{5}{|c|}{$T_{\mathrm{DA}}^{\prime}(\mathrm{meV})$} \\
\hline & $R(\AA)$ & NET-LRC- $\omega$ PBEh & CAM-B3LYP & $\omega \mathrm{B} 97 \mathrm{X}-\mathrm{D}$ & PBE0 & BH\&HLYP \\
\hline \multirow[t]{4}{*}{ Ethylene } & 3.5 & 490.7 & 475.0 & 474.2 & 431.2 & 479.2 \\
\hline & 4.0 & 250.3 & 245.5 & 245.0 & 215.8 & 244.7 \\
\hline & 4.5 & 124.3 & 124.1 & 122.9 & 106.8 & 122.4 \\
\hline & 5.0 & 59.4 & 60.6 & 59.4 & 52.1 & 59.5 \\
\hline \multirow[t]{4}{*}{ Acetylene } & 3.5 & 438.6 & 419.8 & 419.5 & 381.3 & 420.5 \\
\hline & 4.0 & 216.2 & 210.3 & 208.9 & 184.6 & 207.2 \\
\hline & 4.5 & 104.0 & 103.0 & 101.2 & 88.5 & 100.0 \\
\hline & 5.0 & 48.4 & 48.9 & 47.8 & 42.0 & 47.1 \\
\hline \multirow[t]{4}{*}{ Cyclopropene } & 3.5 & 523.4 & 507.5 & 508.0 & 473.0 & 506.8 \\
\hline & 4.0 & 242.7 & 239.2 & 237.9 & 216.0 & 236.1 \\
\hline & 4.5 & 111.0 & 111.1 & 108.3 & 98.0 & 108.5 \\
\hline & 5.0 & 49.7 & 50.6 & 48.6 & 44.3 & 49.0 \\
\hline \multirow[t]{4}{*}{ Cyclobutadiene } & 3.5 & 425.1 & 417.1 & 417.3 & 383.0 & 425.6 \\
\hline & 4.0 & 212.2 & 211.0 & 210.6 & 187.7 & 213.5 \\
\hline & 4.5 & 104.3 & 104.7 & 103.4 & 91.3 & 105.3 \\
\hline & 5.0 & 49.6 & 50.4 & 49.2 & 43.8 & 50.5 \\
\hline \multirow[t]{4}{*}{ Cyclopentadiene } & 3.5 & 436.0 & 433.6 & 433.9 & 396.9 & 440.7 \\
\hline & 4.0 & 216.0 & 217.7 & 217.7 & 192.7 & 219.2 \\
\hline & 4.5 & 105.3 & 107.1 & 105.8 & 92.7 & 107.0 \\
\hline & 5.0 & 49.9 & 51.1 & 49.9 & 44.0 & 50.9 \\
\hline \multirow[t]{4}{*}{ Furane } & 3.5 & NA & 403.1 & 402.7 & 369.0 & 409.7 \\
\hline & 4.0 & NA & 197.7 & 196.4 & 175.1 & 199.0 \\
\hline & 4.5 & NA & 95.2 & 93.3 & 82.5 & 95.0 \\
\hline & 5.0 & NA & 44.8 & 43.6 & 38.6 & 44.5 \\
\hline \multirow[t]{4}{*}{ Pyrrole } & 3.5 & 424.1 & 419.8 & 420.5 & 384.6 & 425.9 \\
\hline & 4.0 & 208.8 & 209.5 & 209.6 & 185.6 & 210.4 \\
\hline & 4.5 & 101.5 & 102.9 & 101.6 & 89.1 & 102.4 \\
\hline & 5.0 & 48.2 & 49.3 & 48.2 & 42.5 & 48.9 \\
\hline
\end{tabular}


Table S3. Electronic coupling energy for hole transfers in the other four cation dimers of the HAB11 set calculated with another three RS and two hybrid density functionals.

\begin{tabular}{llrrrrr}
\hline \hline & \multicolumn{5}{c}{$T_{\text {DA }}^{\prime}(\mathrm{meV})$} \\
\hline \multirow{5}{*}{ Thiophene } & $R(\AA)$ & NET-LRC- $\omega$ PBEh & CAM-B3LYP & $\omega$ B97X-D & PBE0 & BH\&HLYP \\
& 3.5 & 433.0 & 429.9 & 430.1 & 394.4 & 436.2 \\
& 4.0 & 210.7 & 212.0 & 212.3 & 187.8 & 212.8 \\
& 4.5 & 100.7 & 102.4 & 101.4 & 88.4 & 101.8 \\
Imidazole & 5.0 & 46.9 & 48.1 & 47.0 & 41.2 & 47.6 \\
& 3.5 & 402.2 & 397.2 & 397.1 & 363.3 & 403.0 \\
& 4.0 & 194.6 & 194.8 & 193.7 & 172.4 & 195.7 \\
& 4.5 & 93.0 & 94.0 & 92.0 & 81.4 & 93.6 \\
Benzene & 3.0 & 43.5 & 44.4 & 43.1 & 38.3 & 44.1 \\
& 3.5 & $\mathrm{NA}$ & 431.0 & 430.5 & 392.5 & 437.9 \\
& 4.0 & $\mathrm{NA}$ & 211.7 & 211.1 & 185.9 & 212.9 \\
& 4.5 & $\mathrm{NA}$ & 101.7 & 100.2 & 87.0 & 101.4 \\
& 5.0 & $\mathrm{NA}$ & 47.6 & 46.3 & 40.4 & 47.2 \\
Phenol & 3.5 & 346.5 & 349.3 & 347.3 & 317.5 & 348.6 \\
& 4.0 & 162.1 & 165.7 & 164.6 & 145.1 & 172.4 \\
& 4.5 & 75.0 & 77.2 & 75.8 & 65.7 & 80.0 \\
& 5.0 & 34.5 & 35.8 & 34.7 & 30.0 & 37.0 \\
\hline \hline
\end{tabular}


Table S4. Electronic coupling energy for electron transfers in the anion dimers of the HAB7 - set calculated with another three RS and two hybrid density functionals.

\begin{tabular}{|c|c|c|c|c|c|c|}
\hline & & \multicolumn{5}{|c|}{$\overline{T_{\mathrm{DA}}^{\prime}(\mathrm{meV})}$} \\
\hline & $R(\AA)$ & LRC- $\omega$ PBEh & CAM-B3LYP & $\omega \mathrm{B} 97 \mathrm{X}-\mathrm{D}$ & PBE0 & BH\&HLYP \\
\hline \multirow[t]{4}{*}{ Anthracene } & 3.5 & 399.8 & 414.6 & 411.5 & 382.2 & 431.7 \\
\hline & 4.0 & 195.3 & 207.4 & 203.2 & 183.7 & 216.3 \\
\hline & 4.5 & 94.9 & 102.6 & 98.1 & 87.7 & 107.1 \\
\hline & 5.0 & 45.5 & 49.7 & 46.7 & 41.5 & 52.0 \\
\hline \multirow[t]{4}{*}{ Tetracene } & 3.5 & 398.3 & 412.1 & 409.9 & 380.1 & 427.9 \\
\hline & 4.0 & 191.7 & 203.2 & 200.0 & 180.0 & 210.9 \\
\hline & 4.5 & 91.7 & 98.8 & 95.0 & 84.5 & 102.5 \\
\hline & 5.0 & 43.3 & 47.1 & 44.5 & 39.3 & 48.9 \\
\hline \multirow[t]{4}{*}{ Pentacene } & 3.5 & 395.2 & 408.3 & 406.9 & 376.5 & 423.1 \\
\hline & 4.0 & 188.9 & 199.7 & 197.1 & 177.0 & 206.7 \\
\hline & 4.5 & 89.5 & 96.2 & 92.8 & 82.3 & 99.4 \\
\hline & 5.0 & 41.9 & 45.4 & 43.0 & 38.0 & 46.9 \\
\hline \multirow[t]{4}{*}{ Peryfluoroanthracene } & 3.5 & 285.6 & 296.2 & 295.0 & 273.4 & 314.6 \\
\hline & 4.0 & 127.4 & 135.4 & 131.3 & 119.8 & 144.2 \\
\hline & 4.5 & 57.7 & 62.2 & 58.5 & 52.9 & 66.2 \\
\hline & 5.0 & 26.8 & 29.1 & 27.1 & 24.1 & 31.0 \\
\hline \multirow[t]{4}{*}{ Perylene } & 3.5 & 396.6 & 412.0 & 408.6 & 380.0 & 428.8 \\
\hline & 4.0 & 194.7 & 207.3 & 202.7 & 184.0 & 216.1 \\
\hline & 4.5 & 95.4 & 103.4 & 98.6 & 88.9 & 107.9 \\
\hline & 5.0 & 46.1 & 50.5 & 47.2 & 42.5 & 52.8 \\
\hline \multirow[t]{4}{*}{ Perylene Diimide } & 3.5 & 344.3 & 359.3 & 355.5 & 327.8 & 376.3 \\
\hline & 4.0 & 159.6 & 170.7 & 166.3 & 149.5 & 178.8 \\
\hline & 4.5 & 73.3 & 79.6 & 75.5 & 67.3 & 83.4 \\
\hline & 5.0 & 33.5 & 36.7 & 34.2 & 30.3 & 38.5 \\
\hline \multirow[t]{4}{*}{ Porphin } & 3.5 & 351.8 & 364.2 & 362.0 & 335.9 & 379.2 \\
\hline & 4.0 & 165.2 & 174.8 & 172.2 & 155.1 & 182.0 \\
\hline & 4.5 & 77.0 & 82.6 & 79.7 & 71.0 & 85.9 \\
\hline & 5.0 & 35.5 & 38.4 & 36.4 & 32.3 & 40.0 \\
\hline
\end{tabular}


Table S5. Same as Table II but with the aug-cc-pVTZ basis set for heavy atoms.

\begin{tabular}{lcccccc}
\hline \hline Molecule & $\mu$ & $\mathrm{IP}^{(N)}$ & $\mathrm{EA}^{(N)}$ & $\varepsilon_{\mathrm{HO}}^{(N)}$ & $\varepsilon_{\mathrm{HO}}^{(N+1)}$ & $\varepsilon_{\mathrm{LU}}^{(N+1)}$ \\
\hline Anthracene & 0.225 & 7.26 & 0.51 & -7.23 & -0.53 & -0.51 \\
Tetracene & 0.205 & 6.70 & 1.06 & -6.58 & -1.07 & -1.06 \\
Pentacene & 0.190 & 6.31 & 1.44 & -6.30 & -1.46 & -1.44 \\
Peryfluoroanthracene & 0.230 & 8.02 & 1.56 & -8.01 & -1.57 & -1.56 \\
Perylene & 0.210 & 6.78 & 0.94 & -6.77 & -0.97 & -0.92 \\
Perylene Diimide & 0.190 & 7.73 & 2.70 & -7.73 & -2.72 & -2.70 \\
Porphin & 0.225 & 6.93 & 1.48 & -6.89 & -1.56 & -1.42 \\
\hline \hline
\end{tabular}


Table S6. Same as Table V but with the aug-cc-pVTZ basis set for heavy atoms.

\begin{tabular}{|c|c|c|c|c|}
\hline & & \multicolumn{3}{|c|}{$T_{\mathrm{DA}}^{\prime}(\mathrm{meV})$} \\
\hline & $R(\AA)$ & LC-BLYP & B3LYP & Ref. \\
\hline \multirow[t]{5}{*}{ Anthracene } & 3.5 & 411.0 & 379.5 & 421.1 \\
\hline & 4.0 & 214.0 & 188.9 & 212.3 \\
\hline & 4.5 & 112.1 & 94.1 & 106.1 \\
\hline & 5.0 & 59.4 & 47.2 & 52.3 \\
\hline & $\operatorname{MRUE}(\%)$ & 5.6 & 10.5 & - \\
\hline \multirow[t]{5}{*}{ Tetracene } & 3.5 & 401.8 & 375.6 & 417.2 \\
\hline & 4.0 & 203.5 & 182.9 & 204.3 \\
\hline & 4.5 & 102.9 & 88.6 & 97.9 \\
\hline & 5.0 & 52.2 & 42.9 & 45.4 \\
\hline & MRUE(\%) & 6.0 & 8.9 & - \\
\hline \multirow[t]{5}{*}{ Pentacene } & 3.5 & 393.2 & 371.0 & 411.0 \\
\hline & 4.0 & 196.3 & 178.6 & 198.0 \\
\hline & 4.5 & 97.3 & 85.1 & 92.4 \\
\hline & 5.0 & 48.1 & 40.4 & 41.0 \\
\hline & $\operatorname{MRUE}(\%)$ & 7.0 & 7.2 & - \\
\hline \multirow[t]{5}{*}{ Peryfluoroanthracene } & 3.5 & 293.8 & 272.6 & 310.9 \\
\hline & 4.0 & 138.3 & 123.0 & 139.1 \\
\hline & 4.5 & 64.5 & 54.9 & 59.9 \\
\hline & 5.0 & 29.8 & 24.3 & 24.0 \\
\hline & $\operatorname{MRUE}(\%)$ & 9.5 & 8.4 & - \\
\hline \multirow[t]{5}{*}{ Perylene } & 3.5 & 407.5 & 381.1 & 423.7 \\
\hline & 4.0 & 217.0 & 194.3 & 220.7 \\
\hline & 4.5 & 117.7 & 100.4 & 116.6 \\
\hline & 5.0 & 65.3 & 52.8 & 62.8 \\
\hline & MRUE(\%) & 2.6 & 13.0 & - \\
\hline \multirow[t]{5}{*}{ Perylene Diimide } & 3.5 & 343.7 & 327.4 & 373.8 \\
\hline & 4.0 & 170.1 & 156.0 & 179.2 \\
\hline & 4.5 & 84.7 & 74.5 & 84.1 \\
\hline & 5.0 & 42.7 & 36.0 & 38.0 \\
\hline & $\operatorname{MRUE}(\%)$ & 6.6 & 10.5 & - \\
\hline \multirow[t]{5}{*}{ Porphin } & 3.5 & 361.1 & 333.5 & 374.5 \\
\hline & 4.0 & 180.1 & 159.2 & 182.9 \\
\hline & 4.5 & 90.0 & 75.8 & 89.4 \\
\hline & 5.0 & 45.2 & 36.3 & 44.1 \\
\hline & MRUE(\%) & 2.0 & 14.2 & - \\
\hline Total & MRUE(\%) & 5.6 & 10.4 & - \\
\hline
\end{tabular}

